\title{
Inflammatory Scores: Comparison and Utility in HCC Patients Undergoing Transarterial Chemoembolization in a North American Cohort
}

\author{
Shamar Young $\mathbb{D}^{\prime}$ \\ Isa Cam' \\ Mehmet Gencturk' \\ Nathan Rubin $\mathbb{D}^{2}$ \\ Donna D'souza' \\ Siobhan Flanagan' \\ Jafar Golzarian' \\ Tina Sanghvi ${ }^{3}$ \\ 'University of Minnesota, Department of \\ Radiology, Division of Interventional \\ Radiology, Minneapolis, MN, 55455, USA; \\ ${ }^{2}$ Biostatistics Core, Masonic Cancer \\ Center, University of Minnesota, \\ Minneapolis, MN, USA; ${ }^{3}$ Minneapolis VA \\ Medical Center, Department of \\ Radiology, Minneapolis, MN, USA
}

Background: The purpose of this study is to determine and compare the ability of neutrophil-to-lymphocyte ratio (NLR), platelet-to-lymphocyte ratio (PLR), aspartateaminotransferase-to-lymphocyte ratio (ALRI), systemic-inflammation index (SII) and lymphocyte count to predict oncologic outcomes in hepatocellular carcinoma (HCC) patients undergoing transarterial chemoembolization (TACE).

Materials and Methods: A single-center retrospective review of 296 patients who were treated for 457 HCCs was performed. Pre- and post-treatment laboratory and treatment outcome variables were collected. Objective radiologic response (ORR), progression-free survival (PFS), and overall survival (OS) were evaluated. Patients were categorized into above and below median scores and compared.

Results: The median pretreatment NLR, PLR, ALRI, SII, and lymphocyte count were 2.7 (range: 0.4-55), 88.3 (range: 0.1-840), 71.8 (range: 0.1-910), 238.1 (range: 0.1-5150.8), and 1 (range: $0.1-5.2) 10^{3} / \mu \mathrm{L}$, respectively. Patients with above median ALRI scores were less likely to achieve an ORR as compared to those with below median ALRI values (132 (132/ $163,81 \%)$ vs $150(150 / 163,92 \%), p=0.004)$. On univariate analysis, patients with above median pretreatment NLR (HR 1.41, 95\% CI: $1.09-1.83, p=0.01$ ) and below median lymphocyte count (HR $0.69,95 \%$ CI: $0.53-0.92, p=0.01$ ) had significantly worse PFS. The relationship between PFS and NLR $(p=0.08)$ as well as lymphocytes $(p=0.20)$ no longer remained on multivariate analysis. On univariate analysis, below median pretreatment NLR (HR 1.72, 95\% CI: 1.2-2.45, $p=0.003$ ) and ALRI (HR 1.52, 95\% CI: $1.05-2.2) ; p=$ 0.03 ) as well as above median lymphocyte count (HR 0.48, 95\% CI: $0.34-0.7, p<0.0001$ ) were associated with improved OS. The significant relationship between lymphocytes and OS remained on multivariate analysis (HR $0.50,95 \%$ CI: $0.28-0.9, p=0.02$ ), but the relationship with NLR $(p=0.94)$ did not persist.

Conclusion: NLR is predictive of PFS and OS in patients with HCC undergoing TACE and may be superior to other inflammatory scores (PLR, ALRI, and SII) in this setting. However, lymphocyte count may be most predictive of OS.

Keywords: hepatocellular carcinoma, chemoembolization, neutrophil-to-lymphocyte ratio, platelet-to-lymphocyte ratio, aspartate-aminotransferase-to-lymphocyte ratio, systemicinflammation index

\section{Introduction}

Hepatocellular carcinoma (HCC) is the third most common cause of cancer death worldwide $^{1}$, and since the publication of two ground-breaking clinical trials which demonstrated the superiority of transarterial chemoembolization (TACE) to best
Correspondence: Shamar Young University of Minnesota, Department of Radiology, Division of Interventional Radiology, 420 Delaware St SE,

Minneapolis, MN, 55455, USA

Tel +612-624-6189

Email youn1862@umn.edu 
supportive care, TACE has been a mainstay of HCC treatment. ${ }^{2,3}$ As such, TACE is included in HCC treatment algorithms by virtually every major liver/HCC society, including the Barcelona Clinic Liver Cancer, American Association for the Study of Liver Disease, and European Association for the Study of the Liver (EASL) ${ }^{4-6}$ However, there have also been significant advances in the treatment options for patients with HCC over the last several years. This underlies the importance of identifying prognostic factors not only for patient education but also to select the best treatment strategy for each patient.

The tumor microenvironment and immune system have become increasingly recognized as integral components in cancer outcomes. At the same time, systemic inflammation has been recognized as an indicator of poorer outcomes. This has led to the investigation of neutrophil-tolymphocyte ratio (NLR) and platelet-to-lymphocyte ratio (PLR) in the setting of TACE and HCC. ${ }^{7-29}$ While a number of papers have been written on the subject, there has been some disagreement over whether or not NLR and PLR are predictive of meaningful oncologic outcomes. This inconsistency may in part stem from the fact that the majority of studies have come from Asia, where the HCC patient population is not only significantly different from North America and Europe, but the included patients often have very advanced disease. While it is common for studies from Asia to treat patients with relatively large HCCs and those with aggressive features such as portal vein invasion with TACE, these patients are often treated with other means such as radioembolization in different parts of the world. Furthermore, we know that TACE techniques vary significantly between geographical regions. ${ }^{30,31}$ This raises the question as to whether or not PLR and NLR are useful for predicting oncologic outcomes in a North American cohort. The interest in this question is emphasized by the fact that some prior investigations of NLR in North America have failed to show its utility, ${ }^{9}$ while others have indicated it is of benefit, at least in terms of predicting radiologic response. ${ }^{29}$

In addition, other markers of systemic inflammation, such as aspartate aminotransferase-to-lymphocyte ratio (ALRI) and systemic-inflammation index (SII) which have shown promise in HCC patients treated by other means, ${ }^{31-33}$ have not been well studied in HCC patients undergoing TACE. To the authors' knowledge, only 2 studies have investigated the utility of $\mathrm{ALRI}^{27,34}$ and 3 have investigated the utility of $\mathrm{SII}^{27,28,34}$ in this patient population. These papers all originate from Asia and have the same issues when being applied to North American and European patients as were described above.

Finally, lymphocytes, which are integral to all four inflammatory scores described above, have also not been well studied in HCC patients undergoing TACE, despite being shown to be of interest in other settings. ${ }^{35}$ In particular, little data is available which determines if NLR, PLR, ALRI, and SII are superior to simple lymphocyte count. This issue is emphasized by the fact that rarely have all four inflammatory scores and lymphocyte count been applied to the same cohort, making it difficult to compare the relative value of each in predicting outcomes.

Therefore, this study aims to evaluate and compare the ability of NLR, PLR, ALRI, and SII to predict oncologic outcomes in patients with HCC treated by TACE in a North American cohort and further investigate the role of lymphocyte count in these predictions.

\section{Materials and Methods}

After Internal Review Board approval, all consecutive patients treated with TACE at a single center between 1/ 1/2010 and 7/1/2018 were retrospectively reviewed. The patients' electronic medical records were reviewed for preand post-treatment laboratory values, demographic data, and tumor characteristics. Radiologic response was evaluated utilizing the European Association for the Study of the Liver (EASL) criteria. An objective radiologic response (ORR) was considered to be a partial or complete response by EASL criteria. The initial response by EASL was recorded at 1 month post first TACE imaging, while maximal response was recorded in those patients who underwent more than one TACE treatment. The microcatheter position at delivery was then evaluated and recorded as super selective (sub segmental), selective (segmental), minimally selective ( $<$ lobar, $>$ segmental), or nonselective (lobar). Overall survival (OS) was defined as the time between the initial treatment and time of death from any cause or the last clinical follow-up (at which time data were censored). Progression-free survival was defined as time from initial treatment to progression by EASL criteria or death.

NLR was calculated by dividing the neutrophil count by the lymphocyte count. PLR was calculated by dividing the platelet count divided by the lymphocyte count. ALRI was calculated by dividing the aspartate aminotransferase level by the lymphocyte count. Finally, SII was calculated by multiplying the neutrophil and platelet count and 
dividing that number by the lymphocyte count. Pretreatment laboratory values were typically collected on the day of but prior to treatment; however, values collected within 2 weeks of treatment were included. If the patients' HCC was treated multiple times with TACE, only the first treatment was included in the analysis.

\section{Statistical Analysis}

Demographic and clinical measures were summarized descriptively, either per tumor or per patient, as appropriate. Patients were categorized into above and below median groups for each inflammatory marker, consistent with prior studies. ${ }^{8,10,11}$ Chi-square and two sample $t$-tests were used to compare groups for categorical and numeric measures, respectively. Pearson correlation was calculated for the pre-treatment inflammatory scores vs the pre-treatment log alpha fetoprotein (AFP) and lesion size (cm). One-way ANOVA and Tukey's post-hoc tests were used to determine significance in the relationship between cause of cirrhosis and pre-treatment inflammatory scores.

The OS and PFS curves were represented with the Kaplan-Meier method. Cox regression models were used to determine hazard ratios (HR) and statistical significance of the pre-treatment inflammatory above or below median groups. For OS models, only data from the first tumor per patient was included. For PFS models, all tumors per patient were included along with a cluster term to correct the standard errors using the grouped jackknife method that accounts for clustering due to multiple observations per patient. Both univariate models and models adjusting for the other pretreatment inflammatory groups in addition to pre-treatment $\log$ AFP, lesion size $(\mathrm{cm})$, and Child Pugh score were performed. These covariates were chosen a priori due to their clinical relevance. Due to missing data, there were 274 TACE treatments included in the PFS model with all adjustment factors and 170 in the adjusted OS model. $p$-values $<0.05$ were considered statistically significant. R (R Core Team, Version 3.6.0) was used for all statistical analyses.

\section{Results}

In total, 296 patients who were treated for $457 \mathrm{HCCs}$ with TACE were included. Demographic data can be found in Table 1. The cohort consisted of 64 (64/296, 21.6\%) women and $232(232 / 296,78.4 \%)$ men with a mean age of $61.4 \pm 8.2$ years. The most common cause of cirrhosis was hepatitis C (144/296 patients, $48.6 \%)$, and the mean size of treated $\mathrm{HCC}$ was $3.5 \pm 3.4 \mathrm{~cm}$.
Table I Demographic Data

\begin{tabular}{|c|c|}
\hline Variable & Patients $=296, \mathrm{HCCs}=457$ \\
\hline Age (years) & $61.4 \pm 8.2$ \\
\hline Ethnicity & \\
\hline Caucasian/white non-hispanic & 209 (209/296, 70.6\%) \\
\hline Black/African American & 37 (37/296, 12.5\%) \\
\hline Asian & $21(2 \mathrm{I} / 296,7.1 \%)$ \\
\hline Hispanic & $21(2 \mathrm{I} / 296,7.1 \%)$ \\
\hline Native American & $8(8 / 296,2.7 \%)$ \\
\hline Cause of cirrhosis & \\
\hline Hepatitis C & 144 (144/296, 48.6\%) \\
\hline Alcohol & $39(39 / 296,13.2 \%)$ \\
\hline Alcohol and hepatitis C & $36(36 / 296,12.2 \%)$ \\
\hline $\mathrm{NASH}$ & 27 (27/296, 9.1\%) \\
\hline Hepatitis B & $26(26 / 296,8.8 \%)$ \\
\hline Cryptogenic & $17(17 / 296,5.7 \%)$ \\
\hline Other* & $7(7 / 296,2.4 \%)$ \\
\hline Sex & \\
\hline Female & $64(64 / 296,21.6 \%)$ \\
\hline Male & $232(232 / 296,78.4 \%)$ \\
\hline HCC size $(\mathrm{cm})$ & $3.5 \pm 3.4$ \\
\hline Number of TACE treatments & $1.4 \pm 0.7$ \\
\hline Selectivity of TACE treatments & \\
\hline Lobar & $3 \mathrm{I}(3 \mathrm{I} / 457,6.8 \%)$ \\
\hline <Lobar, >segmental & $86(86 / 457,18.8 \%)$ \\
\hline Segmental & $200(200 / 457,43.8 \%)$ \\
\hline Sub-segmental & 140 (140/457, 30.6\%) \\
\hline WBC $\left(10^{9} / \mathrm{L}\right)$ & $5.4 \pm 2.2$ \\
\hline Neutrophils $\left(10^{9} / \mathrm{L}\right)$ & $3.3 \pm 1.9$ \\
\hline Lymphocytes $\left(10^{9} / \mathrm{L}\right)$ & $1.2 \pm 0.7$ \\
\hline Hemoglobin $(g / d L)$ & $12.6 \pm 2.6$ \\
\hline INR & $1.2 \pm 0.2$ \\
\hline Creatinine (mg/dL) & $1 \pm 1$ \\
\hline Total bilirubin (mg/dL) & $\mathrm{I} .3 \pm 0.7$ \\
\hline Albumin $(\mathrm{g} / \mathrm{dL})$ & $3.2 \pm 0.6$ \\
\hline MELD & $10.5 \pm 3.5$ \\
\hline Child-Pugh score & $6.5 \pm 1.3$ \\
\hline ECOG & $0.3 \pm 0.5$ \\
\hline Alpha fetoprotein $(\mathrm{ng} / \mathrm{mL})$ & $28.6(0-363,848.3)$ \\
\hline
\end{tabular}

Notes: *Other causes included hemochromatosis, primary biliary cirrhosis, autoimmune hepatitis, and cystic fibrosis. All numeric measures summarized as mean \pm SD, except for AFP, which was summarized as median (range).

Abbreviations: HCC, hepatocellular carcinoma; NASH, non-alcoholic steatohepatitis; TACE, transarterial chemoembolization; $\mathrm{cm}$, centimeter; ECOG, Eastern Cooperative Oncology Group; WBC, white blood cell count.

Table 2 provides summaries for the pretreatment NLR, PLR, ALRI, SII and lymphocyte count values. The median pretreatment NLR, PLR, ALRI, SII, and lymphocyte count were 2.7 (range: 0.4-55), 88.3 (range: 0.1-840), 71.8 
Table 2 Descriptive Summaries for the Pre- and Post-Treatment Inflammatory Values

\begin{tabular}{|c|c|}
\hline Variable & \\
\hline \multicolumn{2}{|l|}{ Pretreatment NLR } \\
\hline Mean & $4 \pm 4.8$ \\
\hline Median & $2.7(0.4,55)$ \\
\hline \multicolumn{2}{|l|}{ Pretreatment PLR } \\
\hline Mean & $118.8 \pm 110.3$ \\
\hline Median & $88.3(0.1,840.0)$ \\
\hline \multicolumn{2}{|l|}{ Pretreatment ALRI } \\
\hline Mean & $111.1 \pm 133.5$ \\
\hline Median & $71.8(0.1,910.0)$ \\
\hline \multicolumn{2}{|l|}{ Pretreatment SII } \\
\hline Mean & $442.2 \pm 606.1$ \\
\hline Median & $238.1(0.1,5150.8)$ \\
\hline \multicolumn{2}{|l|}{ Pretreatment lymphocyte count $\left(10^{3} / \mu \mathrm{L}\right)$} \\
\hline Mean & $1.2 \pm 0.7$ \\
\hline Median & $\mathrm{I}(0.1,5.2)$ \\
\hline
\end{tabular}

Abbreviations: NLR, neutrophil-to-lymphocyte ratio; PLR, platelet-to-lymphocyte ratio; ALRI, aspartate-aminotransferase-to-lymphocyte ratio; SII, systemicinflammatory index.

(range: 0.1-910), 238.1 (range: 0.1-5150.8), and 1 (range: $0.1-5.2) 10^{3} / \mu \mathrm{L}$, respectively. Supplemental Figure 1 evaluates the relationship between the pretreatment inflammatory scores and the pretreatment log AFP. However, log AFP did not correlate with inflammatory scores. Supplemental Figure 2 evaluates the relationship between the pretreatment inflammatory scores and the pretreatment tumor size. While there was no correlation with tumor size in regard to NLR and ALRI, there was a modest correlation between PLR $(r=0.13, p<0.001)$ and SII $(r=0.17$, $\mathrm{p}<0.001)$. Supplemental Figure 3 evaluates the relationship between the cause of cirrhosis and inflammatory scores. Again, there was not a strong correlation between inflammatory scores and the underlying cause of cirrhosis. However, cryptogenic cirrhosis had significantly higher pre-treatment PLR and SII than all other causes (all $p$ values $<0.025$ after Tukey adjustment).

\section{Radiologic Response}

Data on the ability of inflammatory scores and lymphocyte count to predict the radiologic response can be found in Table 3. Of note patients with above median ALRI were less likely to achieve an ORR than those below the median $(132(132 / 163,81 \%)$ vs $150(150 / 163$, $92 \%), p=0.004)$.

\section{Progression-Free Survival}

Progression-free survival Kaplan-Meier curves for median pretreatment NLR, PLR, ALRI, and SII can be found in Figure 1. This showed that patients with above median pretreatment NLR (HR 1.41, 95\% CI: $1.09-1.83, p=0.01$ ) and below median lymphocyte count (HR 0.69, 95\% CI: 0.53-0.92, $p=0.01$ ) had significantly worse PFS. However, neither median pretreatment PLR (HR 1.12, 95\% CI: 0.85-1.48, $p=0.43$ ), ALRI (HR 1.11, 95\% CI: $0.83-1.48, p=0.47$ ), nor SII (HR 1.13, 95\% CI: 0.85-1.5, $p=0.41)$ predicted PFS.

\section{Cox Regression Analysis Using NLR, PLR, ALRI, and SII}

In a Cox regression model which included all pretreatment inflammatory scores it was found that pretreatment NLR (HR 1.48, 95\% CI: 1.10-1.99, $p=0.009$ ) was associated with PFS while PLR (HR 0.97, 95\% CI: 0.68-1.37, $p=0.86$ ), ALRI (HR 0.95, 95\% CI: 0.70-1.30, $p=0.77$ ) and SII (HR 0.99, 95\% CI: 0.70-1.39, $p=0.95$ ) were not. If tumor size, log AFP, and Child-Pugh classification were added to this model, pretreatment NLR remained a significant predictor of PFS (HR 1.50, 95\% CI: $1.08-$ 2.10, $p=0.017$ ). While pretreatment PLR (HR 0.94, 95\% CI: $0.62-1.44, p=0.79$ ), ALRI (HR 0.79 , 95\% CI: $0.56-$ $1.11, p=0.18$ ), and SII (HR 1.0, 95\% CI: 0.66-1.51, $p=0.99$ ) were not. Pretreatment AFP (HR 1.08, 95\% CI: $1.01-1.16, p=0.034)$ was found to correlate with PFS, while tumor size (HR 0.98, 95\% CI: 0.92-1.04, $p=0.46$ ) and Child-Pugh (HR 1.11, 95\% CI: 0.97-1.28, $p=0.14$ ) did not.

\section{Cox Regression Analysis Using NLR, PLR, ALRI, SII, and Lymphocyte Count}

In a Cox regression model which included all pretreatment inflammatory scores as well as lymphocyte count, it was found that neither NLR (HR 1.28, 95\% CI: 0.90-1.82, $p=$ 0.17), PLR (HR 0.91, 95\% CI: $0.64-1.3, p=0.61$ ), ALRI (HR 0.88, 95\% CI: 0.65-1.2, $p=0.43$ ), SII (HR 1.05, 95\% CI: $0.75-1.48, p=0.77$ ), or lymphocyte count (HR 0.73, 95\% CI: $0.49-1.09, p=0.12$ ) were correlated with PFS. If tumor size, log AFP, and Child-Pugh classification were added to this model, again neither pretreatment NLR (HR 1.27, 95\% CI: 0.85-1.91, $p=0.25$ ), PLR (HR 0.88, 95\% CI: $0.58-1.32, p=0.53$ ), ALRI (HR 0.75, 95\% CI: $0.53-$ $1.05, p=0.09$ ), SII (HR 1.07, 95\% CI: 0.71-1.61, $p=0.76$ ) or lymphocyte count (HR $0.70,95 \%$ CI: $0.47-$ 
Table 3 Radiologic Response by Inflammatory Markers. Comparison Between Treated Lesions with Above and Below Average (Log Mean and Median)

\begin{tabular}{|c|c|c|c|}
\hline Variable & Above Median & Below Median & $p$ value \\
\hline \multicolumn{4}{|c|}{ Pretreatment NLR $\mathrm{n}=357$} \\
\hline \multicolumn{4}{|l|}{ Initial response } \\
\hline $\mathrm{CR}$ & $102(102 / 179,57 \%)$ & $90(90 / 178,50.6 \%)$ & 0.43 \\
\hline $\mathrm{PR}$ & $45(45 / 179,25.1 \%)$ & 53 (53/178, 29.8\%) & \\
\hline SD & $24(24 / 179,13.4 \%)$ & 30 (30/I78, 16.8\%) & \\
\hline PD & 8 (8/I79, 4.5\%) & $5(5 / 178,2.8 \%)$ & \\
\hline ORR & I47 (I47/I79, 82.1\%) & I43 (I43/I78, 80.4\%) & 0.67 \\
\hline \multicolumn{4}{|c|}{ Maximal response } \\
\hline $\mathrm{CR}$ & $112(112 / 179,62.6 \%)$ & $113(113 / 178,63.5 \%)$ & 0.95 \\
\hline$P R$ & 43 (43/I79, 24\%) & $42(42 / 178,23.6 \%)$ & \\
\hline SD & 17 (I7/I79, 9.5\%) & $18(18 / 178,10.1 \%)$ & \\
\hline PD & 7 (7/I79, 3.9\%) & $5(5 / 178,2.8 \%)$ & \\
\hline ORR & 155 (I55/I79, 86.6\%) & 155 (I55/I78, 87.1\%) & 0.89 \\
\hline \multicolumn{4}{|c|}{ Pretreatment PLR $n=343$} \\
\hline \multicolumn{4}{|l|}{ Initial response } \\
\hline $\mathrm{CR}$ & 87 (87/I7I, 50.9\%) & 98 (98/I72, 57\%) & 0.15 \\
\hline PR & 54 (54/I7I, 3I.6\%) & 40 (40/I $72,23.3 \%)$ & \\
\hline SD & 22 (22/I7I, I2.9\%) & $30(30 / 172,17.4 \%)$ & \\
\hline PD & 8 (8/I7I, 4.6\%) & $4(4 / 172,2.3 \%)$ & \\
\hline ORR & $|4|(|4| /|7|, 82.5 \%)$ & $138(138 / 172,80.3 \%)$ & 0.60 \\
\hline \multicolumn{4}{|c|}{ Maximal response } \\
\hline $\mathrm{CR}$ & 100 (100/I7I, 58.5\%) & $116(116 / 172,67.4 \%)$ & 0.09 \\
\hline PR & 49 (49/I7I, 28.7\%) & 33 (33/172, 19.2\%) & \\
\hline SD & I4 (|4/I7I, 8.2\%) & 19 (19/172, 11\%) & \\
\hline PD & 8 (8/I7I, 4.7\%) & $4(4 / 172,2.4 \%)$ & \\
\hline ORR & I49 (|49/I7I, 87.2\%) & 149 (149/172, 86.6\%) & 0.89 \\
\hline \multicolumn{4}{|c|}{ Pretreatment ALRI $n=326$} \\
\hline \multicolumn{4}{|l|}{ Initial response } \\
\hline $\mathrm{CR}$ & $89(89 / 163,54.6 \%)$ & $89(89 / 163,54.6 \%)$ & 0.36 \\
\hline PR & $38(38 / 163,23.3 \%)$ & $48(48 / 163,29.4 \%)$ & \\
\hline SD & $28(28 / 163,17.2 \%)$ & $22(22 / 163,13.5 \%)$ & \\
\hline PD & $8(8 / 163,4.9 \%)$ & $4(4 / 163,2.5 \%)$ & \\
\hline ORR & $127(127 / 163,77.9 \%)$ & 137 (137/163, 84\%) & 0.14 \\
\hline \multicolumn{4}{|c|}{ Maximal response } \\
\hline $\mathrm{CR}$ & $104(104 / 163,63.8 \%)$ & $106(106 / 163,65 \%)$ & 0.01 \\
\hline PR & $28(28 / 163,17.2 \%)$ & $44(44 / 163,27 \%)$ & \\
\hline SD & $23(23 / 163,14.1 \%)$ & $10(10 / 163,6.1 \%)$ & \\
\hline PD & $8(8 / 163,4.9 \%)$ & $3(3 / 163,1.9 \%)$ & \\
\hline ORR & $132(132 / 163,81 \%)$ & $150(150 / 163,92 \%)$ & 0.004 \\
\hline \multicolumn{4}{|c|}{ Pretreatment SII n = 342} \\
\hline \multicolumn{4}{|l|}{ Initial response } \\
\hline $\mathrm{CR}$ & 93 (93/17I, 54.4\%) & 91 (9I/I7I, 53.2\%) & 0.62 \\
\hline$P R$ & 49 (49/I7I, 28.7\%) & 45 (45/I7I, 26.3\%) & \\
\hline SD & $22(22 / 17|| 2.9 \%)$, & 30 (30/I7I, 17.5\%) & \\
\hline PD & 7 (7/I7I, 4\%) & $5(5 / 171,3 \%)$ & \\
\hline
\end{tabular}

(Continued) 
Table 3 (Continued).

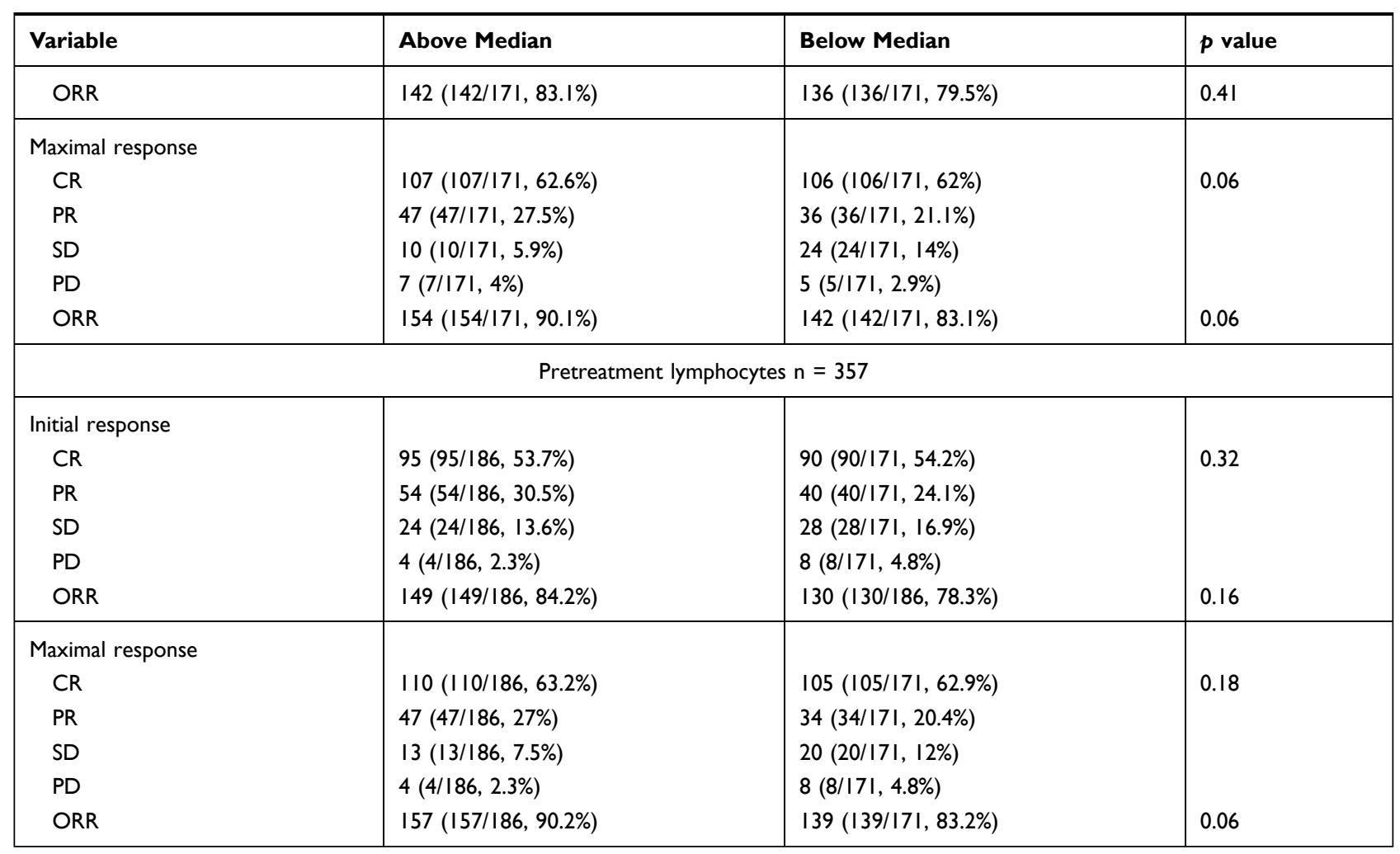

Abbreviations: NLR, neutrophil-to-lymphocyte ratio; CR, complete response; PR, partial response; SD, stable disease; PD, progressive disease; ORR, objective radiologic response; PLR, platelet-to-lymphocyte ratio; ALRI, aspartate-aminotransferase-to lymphocyte ratio; SII, systemic-inflammatory index.

1.06, $p=0.09$ ) demonstrated significant association. While AFP (HR 1.08, 95\% CI: 1.00-1.16, $p=0.05$ ) nearly reached significance, neither tumor size (HR 0.98, 95\% CI: 0.93-1.04, $p=0.55$ ) nor Child-Pugh (HR 1.08, 95\% CI: 0.94-1.24, $p=0.28$ ) was associated with PFS.

\section{Overall Survival}

Overall survival Kaplan-Meier curves for median pretreatment NLR, PLR, ALRI, and SII can be found in Figure 2. Those with above median NLR (HR 1.72, 95\% CI: $1.2-$ $2.45) ; p=0.003$ ) and ALRI (HR 1.52, 95\% CI: 1.05-2.2); $p=0.03$ ) had a higher risk of death. Those patients with below median lymphocytes (HR 0.48, 95\% CI: 0.34-0.7, $p<0.0001)$ also had a higher risk of death. There was no statistically significant difference seen in PLR (HR 1.19, 95\% CI: $0.84-1.69, p=0.33$ ) or SII (HR 1.34, 95\% CI: $0.94-1.91, p=0.11)$.

\section{Cox Regression Analysis Using NLR, PLR, ALRI, and SII}

In a Cox regression model which included all pretreatment inflammatory scores, it was found that neither pretreatment
NLR (HR 1.50, 95\% CI: 0.94-2.40, $p=0.09$ ), PLR (HR 0.89, 95\% CI: 0.53-1.51, $p=0.67$ ), ALRI (HR 1.36, 95\% CI: $0.90-2.06, p=0.15$ ) and SII (HR 1.23 95\% CI: $0.70-$ 2.16, $p=0.47$ ) were significantly associated with OS. If tumor size, log AFP, and Child-Pugh classification were added to this model, pretreatment NLR (HR 1.31, 95\% CI: 0.77-2.23, $p=0.32$ ), PLR (HR 1.06, 95\% CI: 0.59-1.89, $p=0.85$ ), ALRI (HR 0.79, 95\% CI: 0.48-1.31, $p=0.36$ ), and SII (HR 1.06, 95\% CI: 0.57-1.97, $p=0.84$ ) were still not significant predictors of OS. Pretreatment AFP (HR 1.05, 95\% CI: $0.98-1.16, p=0.14$ ) and tumor size (HR 1.02, 95\% CI: $0.95-1.09, p=0.62$ ) were not found to be associated with OS; however, Child-Pugh (HR 1.51, 95\% CI: $1.27-1.79, p<0.001)$ did demonstrate an association.

\section{Cox Regression Analysis Using NLR, PLR, ALRI, SII, and Lymphocyte Count}

In a Cox regression model which included all pretreatment inflammatory scores and lymphocyte count, it was found that only pretreatment lymphocytes (HR 0.51, 95\% CI: $0.31-0.86, p=0.010$ ) was significantly associated with OS, while NLR (HR 1.13, 95\% CI: 0.67-1.91, $p=0.64$ ), 
A Progression Free Survival by Pretreatment NLR
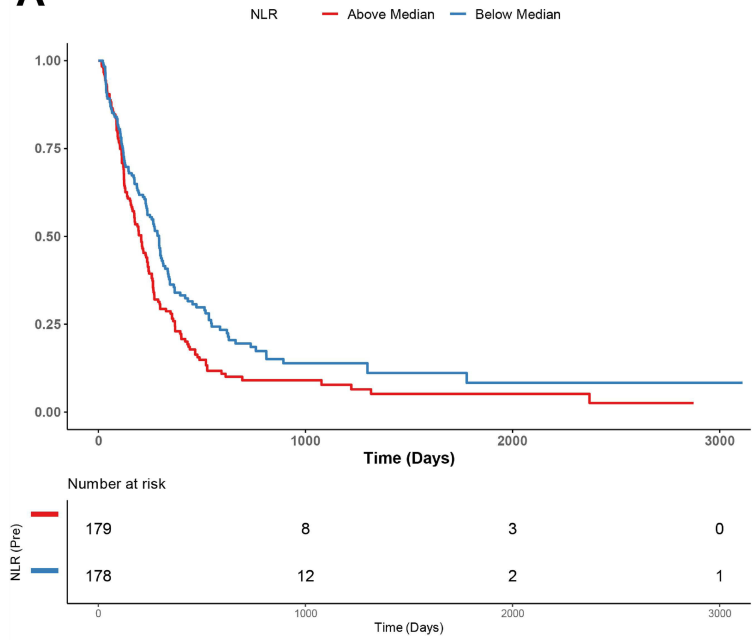

C

Progression Free Survival by Pretreatment ALRI

ALRI - Above Median - Below Median
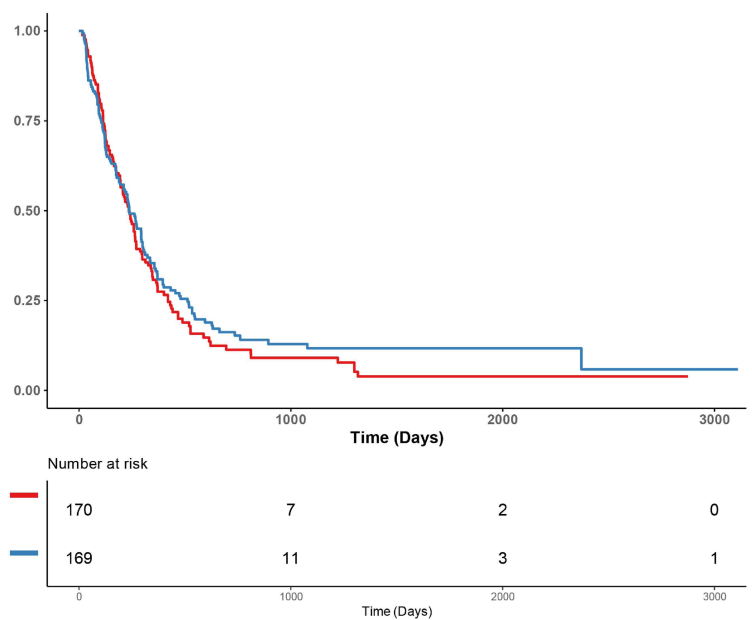

\section{E}

Progression Free Survival by Pretreatment Lymphocytes

Lymphocytes - Above Median - Below Median
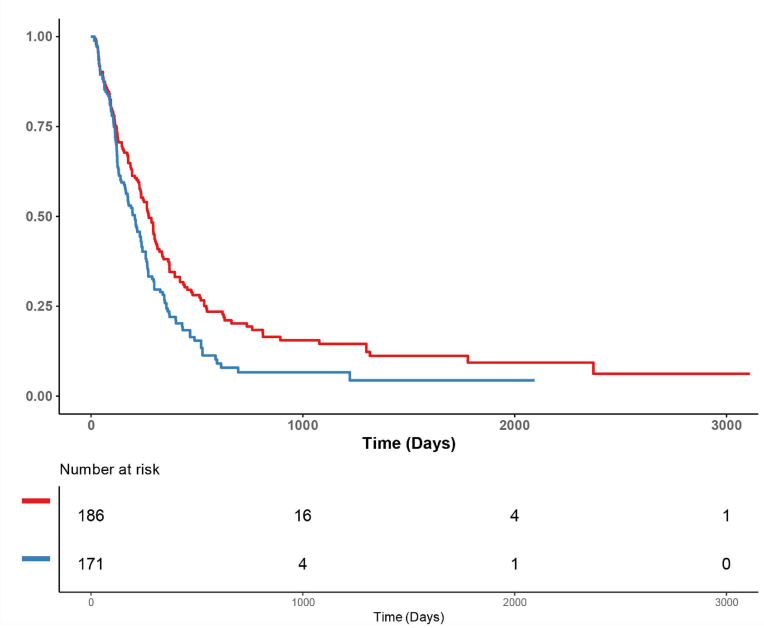

B Progression Free Survival by Pretreatment PLR
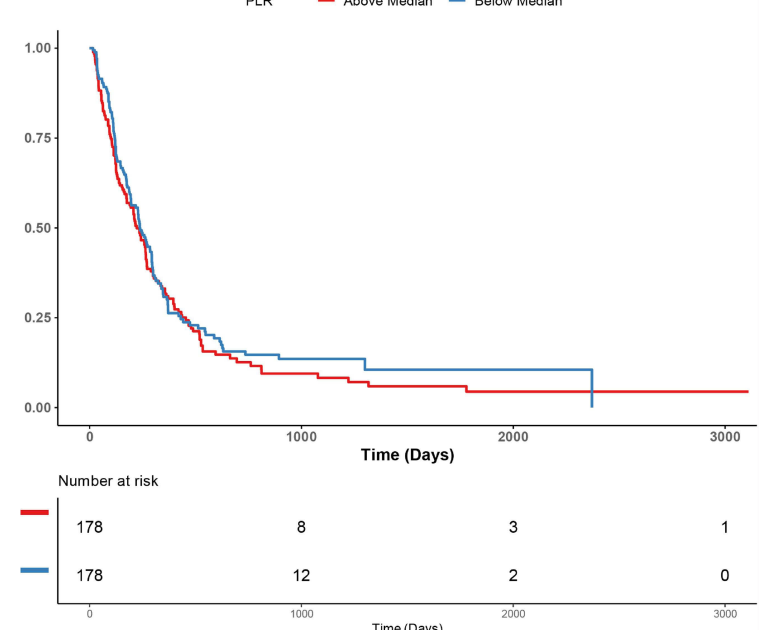

D Progression Free Survival by Pretreatment SII

SII $\quad-$ Above Median - Below Median
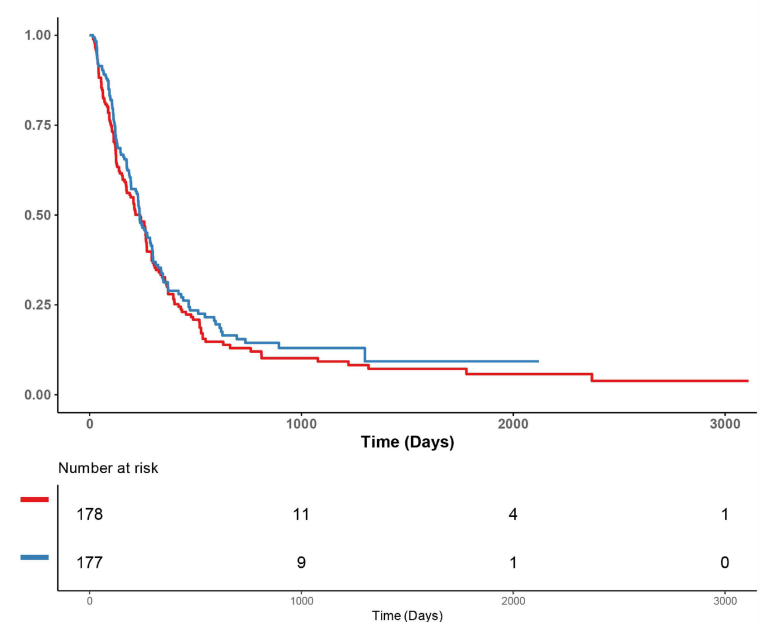

Figure I Kaplan-Meier curves demonstrating progression-free survival (PFS) in patients with above or below median neutrophil-to-lymphocyte ratio (NLR) (A), platelet-tolymphocyte ratio (PLR) (B), aspartate-aminotransferase-to-lymphocyte ratio (ALRI) (C) and systemic-inflammation index (SII) (D) Lymphocyte count (E) . 

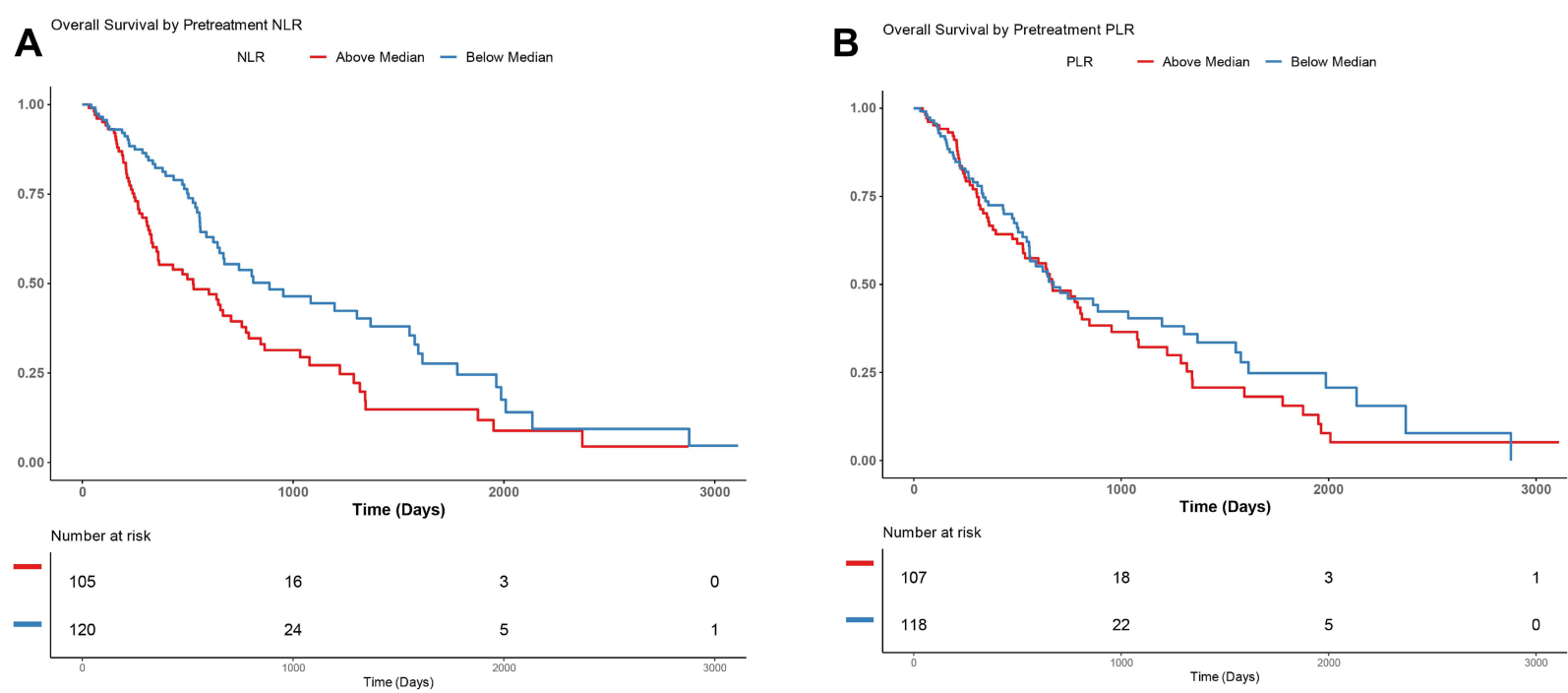

\section{C}

Overall Survival by Pretreatment ALRI

ALRI $\quad$ - Above Median - Below Median

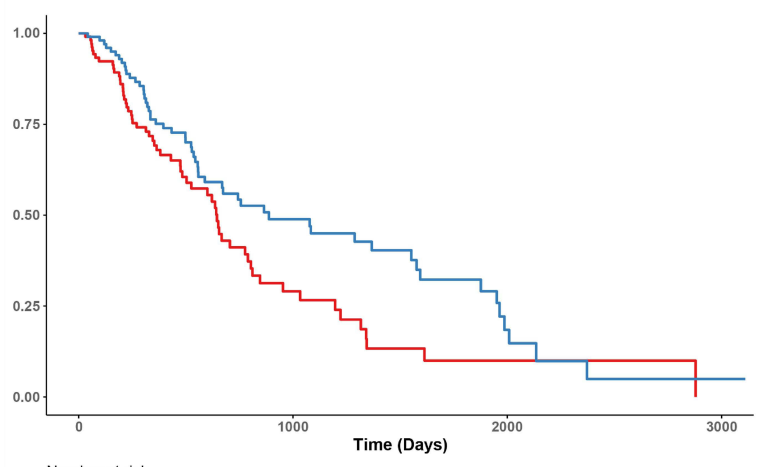

D Overal Survival by Pretreatment SII
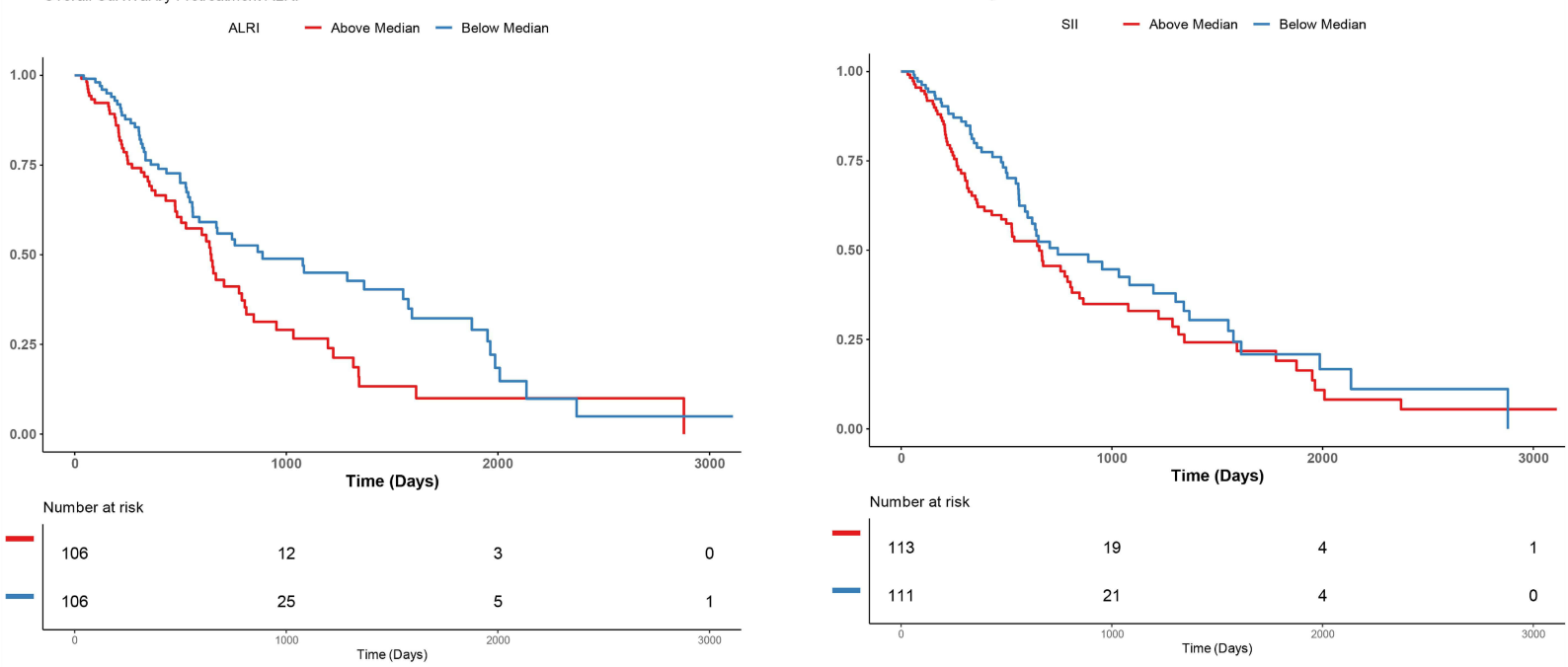

E Overall Survival by Pretreatment Lymphocytes
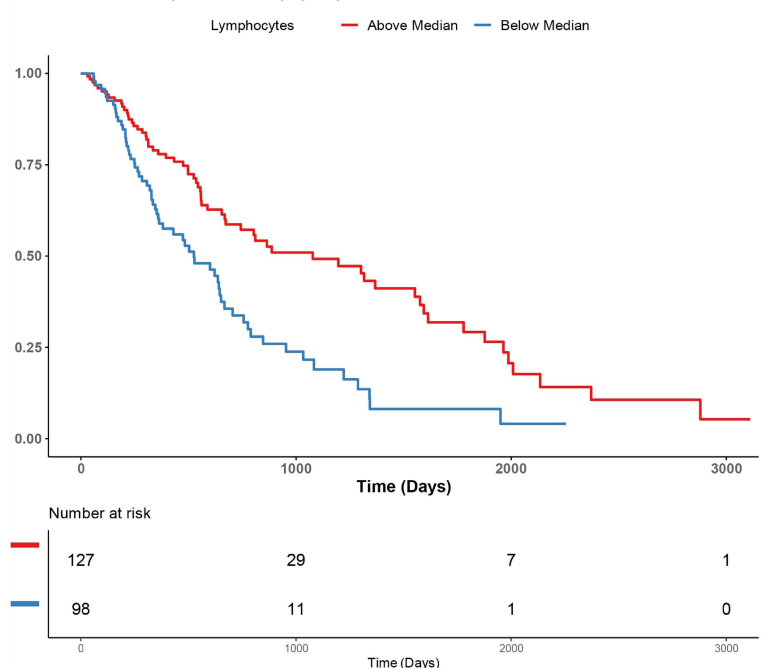

Figure 2 Kaplan-Meier curves demonstrating overall survival (OS) in patients with above or below median neutrophil-to-lymphocyte ratio (NLR) (A), platelet-tolymphocyte ratio (PLR) (B), aspartate-aminotransferase-to-lymphocyte ratio (ALRI) (C) and systemic-inflammation index (SII) (D) Lymphocyte count (E) . 
PLR (HR 0.78, 95\% CI: 0.46-1.32, $p=0.36$ ), ALRI (HR 1.11, 95\% CI: $0.71-1.73, p=0.66$ ), and SII (HR 1.47, 95\% CI: $0.83-2.59, p=0.19)$ were not. If tumor size, $\log$ AFP, and Child-Pugh classification were added to this model, again only pretreatment lymphocyte count (HR $0.50,95 \%$ CI: $0.28-0.9, p=0.02$ ) was significantly associated. Pretreatment NLR (HR 0.98, 95\% CI: 0.54-1.77, $p=0.94$ ), PLR (HR 0.87, 95\% CI: $0.48-1.56, p=0.64$ ), ALRI (HR 0.67, 95\% CI: $0.40-1.13, p=0.13$ ), and SII (HR 1.32, 95\% CI: $0.70-2.50, p=0.39$ ) were not associated. Pretreatment AFP (HR 1.08, 95\% CI: 1.00-1.17, $p=0.06$ ) and tumor size (HR 1.01, 95\% CI: $0.95-1.08, p=$ 0.73) were not associated, while Child-Pugh (HR 1.41, $95 \%$ CI: $1.18-1.70, p<0.001)$ was associated with OS.

\section{Discussion}

As the treatment options for patients with hepatocellular carcinoma (HCC) continue to expand, the importance of finding pre-treatment predictors of outcomes is emphasized. One area which has been investigated for these purposes is inflammatory scores. A number of inflammatory scores have been evaluated, and it is difficult to know which is best for a specific cancer and treatment method or if they provide more information than lymphocyte count alone. This study evaluated four of the commonly used inflammatory scores (NLR, PLR, ALRI, and SII) and pretreatment lymphocyte count in HCC patients undergoing TACE.

Of surprise, radiologic response was poorly predicted by the majority of the inflammatory scores and lymphocyte count. This seems to be in contradiction to Schobert et al who did find a correlation between higher NLR and PLR values and radiologic response when using qEASL. ${ }^{26}$ The results also differ from Cruz et al who showed that those patients with PD immediately after treatment had a significantly higher NLR. ${ }^{29}$ The reason for the discrepancies may be explained by the smaller cohorts in the prior studies as compared to this study. Similarly, of interest, the median of pre-treatment ALRI did show some promise in accurately predicting the maximal response of HCC lesions after TACE. While the reason for ALRI demonstrating promise while other markers did not is not evaluated in this study, it may be related to the fact that ALRI takes into account the aspartate aminotransferase, which may increase this marker's sensitivity for the inflammatory environment of the liver, whereas NLR, PLR, SII, and lymphocyte count may be more tied to the systemic inflammatory environment.
This study also looked at PFS and found that patients with above or below median PLR, ALRI, or SII did not have significantly different PFS. However, those with above median NLR (HR 1.41, 95\% CI: $1.09-1.83, p=$ 0.01 ) and below median lymphocyte count (HR $0.69,95 \%$ CI: $0.53-0.92, p=0.01$ ) had significantly worse PFS on univariate analysis. When a multivariate model which did not include lymphocyte count was created, NLR remained significantly associated, even when adding in other factors such as tumor size, AFP, and Child-Pugh score. However, when lymphocyte count was added to these two multivariate modeling scenarios, neither NLR nor pretreatment lymphocyte count continued to be associated with PFS. When taken as a whole, the findings suggest that NLR and pretreatment lymphocyte count may be superior in terms of predicting those patients who will do poorly following TACE as compared to PLR, ALRI, and SII. The findings also suggest that the predictive ability of NLR may be heavily associated with lymphocyte count, perhaps more so than neutrophil count. The mechanism which connects an elevated NLR, lymphocyte count and poor outcomes in HCC patients is not well understood. However, high NLR and low lymphocyte counts can correlate with depleted lymphocytes, and lymphocytes play a role in cytotoxic cell death and the production of anti-tumor cytokines. ${ }^{36}$ Therefore, lymphopenia is associated with an impaired host immune response to the tumor and thus poor cancerspecific survival. ${ }^{37}$ Systemic neutrophilia has been associated with greater infiltration of tumor-associated macrophages (TAMs) as well as elevated pro-inflammatory cytokines, which promote angiogenesis and tumor growth. ${ }^{38,39}$ The measurement of the relative depletion of lymphocytes and/or neutrophilia likely explains NLR's predictive values, while the lymphocyte role in anti-tumoral responses likely explains lymphocyte count predictive value. The findings of this study suggest that pretreatment NLR and lymphocyte count may allow providers to council patients more accurately on their prognosis.

This study also evaluated the ability of inflammatory scores to evaluate OS and showed that median NLR (HR 1.72, 95\% CI: $1.2-2.45, p=0.003$ ), ALRI (HR 1.52, 95\% CI: $1.05-2.2 ; p=0.03$ ), and lymphocyte count (HR 0.48, 95\% CI: $0.34-0.7, p<0.0001)$ were associated with OS on univariate Cox regression analysis. When multivariate analysis was performed and lymphocyte count was not included, NLR showed a trend toward association with OS in a model that did not include tumor size, AFP, or 
Child-Pugh score, but the trend no longer existed when these variables were included. When lymphocyte count was added to these models, NLR was not significant in either of them, but lymphocyte count was associated with OS in both models. The fact that when lymphocyte count was included as a variable in this study, the correlation between NRL and OS seemed to be negatively impacted is of interest. This would suggest that lymphocyte count was the driving force behind NLRs association with OS and perhaps it would be the best score for predicting OS. To the authors' knowledge, this has not been studied in HCC patients undergoing TACE; however, lymphopenia is known to be associated with poorer outcomes in other settings. ${ }^{35}$

As discussed previously, the majority of studies that have evaluated inflammatory scores in this setting originate from Asia. These studies often differ from North American and European studies in the primary underlying cause of cirrhosis as well as the mean size of tumor. When looking at previous studies from North America and Europe, our findings are consistent with those of Rebonato et al who found that NLR was predictive of OS in their smaller study of 72 patients. ${ }^{14}$ However, they differ from the findings of Sullivan et al who reviewed outcomes in 75 HCC patients treated with surgical resection, liver transplant, or TACE which did not show NLR to be predictive of OS. Furthermore, the multivariate analysis in this relatively larger study would suggest that NLR has a higher association with OS than either PLR, ALRI, or SII while calling into question the importance of any inflammatory scores as compared to other well-established predictive factors such as Child-Pugh score. The question of importance of inflammatory scores is furthered by the fact that when lymphocyte count was added to the multivariate models, lymphocyte count remained associated with OS, while no inflammatory score did.

This study shares similarities with Yang et al who reviewed 189 patients treated with TACE for HCC and evaluated the prognostic value of NLR, PLR, ALRI, and SII. ${ }^{27}$ However, their study differed significantly in the demographics, with 53 patients $(53 / 189,28 \%)$ having tumors greater than $5 \mathrm{~cm}$ and $59(59 / 189,31.2 \%)$ having portal vein thrombosis. The Yang et al's study found that while NLR, PLR, ALRI, and SII were all significantly associated with OS in univariate analysis, only ALRI and SII continued to be associated on multivariate analysis. They also found that there was a correlation between tumor size and portal vein thrombosis and elevated
ALRI and SII. ${ }^{27}$ Of note, Yang et al did not evaluate the effect of lymphocyte count on outcomes, as was done here. Furthermore, the current study found that when separating patients by those with above and below median ALRI, those with lower ALRI scores had significantly longer survival on univariate analysis. However, SII had no significant association with OS in either evaluation. When performing multivariate analysis which included all inflammatory scores, neither ALRI nor SII was predictive of OS. These differences may result from the significantly smaller tumors without portal vein invasion which comprised this study's patient population. This theory is strengthened by the finding in the Yang et al's study that SII and ALRI were correlated with tumor size and portal vein thrombosis, while this study did not find a similar association.

This study has a number of limitations, including its retrospective study design. While a fairly large cohort was included, it is still limited and this is furthered by the fact that not all values were available prior to all treatments. Additionally, the study represents a single quaternary referral center experience and therefore may not be applicable to all practice settings.

In conclusion, while NLR and ALRI are associated with OS in HCC patients undergoing TACE, this study would indicate that NLR has the highest correlation with both OS and PFS of the four inflammatory scores studied. However, these associations are weakened when lymphocyte count is considered and lymphocyte count may be the best predictor of OS. Therefore, NLR and lymphocyte count would likely be the best choice for further investigation in similar patient populations. Furthermore, those patients presenting with elevated NLRs or lymphopenia may benefit from counseling regarding the prognostic implications.

\section{Abbreviations}

ALRI, aspartate-aminotransferase-to-lymphocyte ratio; HCC, hepatocellular carcinoma; NLR, neutrophil-tolymphocyte ratio; ORR, objective radiological response; OS, overall survival; PFS, progression-free survival; SII, systemic-inflammation index; TACE, transarterial chemoembolization.

\section{Ethics Approval and Informed Consent}

The University of Minnesota Institutional Review Board approved this retrospective study. While consent was 
obtained for the procedure, the IRB waived consent for this study, given all patients are asked to opt in or out of research at our institution. This study was completed in accordance with the Declaration of Helsinki. The researchers have taken every precaution to protect the information provided by patients from violating their privacy.

\section{Consent for Publication}

SY gave consent for publication on behalf of all authors.

\section{Acknowledgment}

Research reported in this publication was supported by NIH grant P30CA077598 utilizing the Biostatistics and Bioinformatics Core shared resource of the Masonic Cancer Center, University of Minnesota, and by the National Center for Advancing Translational Sciences of the National Institutes of Health Award Number UL1TR002494. The content is solely the responsibility of the authors and does not necessarily represent the official views of the National Institutes of Health.

\section{Author Contributions}

All authors contributed to data analysis, drafting or revising the article, have agreed on the journal to which the article will be submitted, gave final approval of the version to be published, and agreed to be accountable for all aspects of the work.

\section{Funding}

No funding was received for this article.

\section{Disclosure}

JG is a consultant for Guerbet. SY is a consultant for Boston Scientific. The authors report no other conflicts of interest in this work.

\section{References}

1. Torre LA, Siegel RL, Ward EM, Jemal A. Global cancer incidence and mortality rates and trends-an update. Cancer Epidemiol Prev Biomarkers. 2016;25(1):16-27. doi:10.1158/1055-9965.EPI-15-0578

2. Llovet JM, Real MI, Montana X, et al. Arterial embolization or chemoembolization versus symptomatic treatment in patients with unresectable hepatocellular carcinoma: a randomized controlled trial. Lancet. 2002;359:1734-1739. doi:10.1016/S0140-6736(02)08649-X

3. Lo CM, Ngan H, Tso WK, et al. Randomized controlled trial of transarterial lipiodol chemoembolization for unresectable hepatocellular carcinoma. Hepatology. 2002;35:1164-1171. doi:10.1053/ jhep. 2002.33156

4. Forner A, Gilabert M, Bruix J, Raoul JL. Treatment of intermediate-stage hepatocellular carcinoma. Nat Rev Clin Oncol. 2014;11:525-535. doi:10.1038/nrclinonc.2014.122
5. Heimbach J, Kulik LM, Finn R, et al. AASLD Guidelines for the treatment of hepatocellular carcinoma. Hepatology. 2017. doi:10.1002/hep.29086

6. Llovet JM, Ducreux M, Lencioni R, et al. EASL-EORTC clinical practice guidelines: management of hepatocellular carcinoma. J Hepatol. 2012;56:908-943.

7. Li S, Feng X, Cao G, Wang Q, Wang L. Prognostic significance of inflammatory indices in hepatocellular carcinoma treated with transarterial chemoembolization: a systematic review and meta-analysis. PLoS One. 2020;15(6):e0230879. doi:10.1371/journal.pone.0230879

8. Wang C, Wang M, Zhang X, et al. The neutrophil-to-lymphocyte ratio is a predictive factor for the survival of patients with hepatocellular carcinoma undergoing transarterial chemoembolization. Ann Transl Med. 2020;8(8):541. doi:10.21037/atm.2020.02.113

9. Sullivan K, Groeschl R, Turaga K, et al. Neutrophil-to-lymphocyte ratio as a predictor of outcomes for patients with hepatocellular carcinoma: a Western perspective. J Surg Oncol. 2014;109 (2):95-97. doi:10.1002/jso.23448

10. Wang H, Lin C, Fan W, et al. Dynamic changes in the neutrophil-tolymphocyte ratio predict the prognosis of patients with hepatocellular carcinoma undergoing transarterial chemoembolization. Cancer Manag Res. 2020;12:3433-3444. doi:10.2147/CMAR.S245396

11. Tian X-C, Liu X-L, Zeng F-R, Chen Z, Wu D-H. Platelet-tolymphocyte ratio acts as an independent risk factor for patients with hepatitis B virus-related hepatocellular carcinoma who received transarterial chemoembolization. Eur Rev Med Pharmcol Sci. 2016;20:2302-2309.

12. Xu X, Chen W, Zhang L, et al. Prognostic significance of neutrophil to lymphocyte ratio in patients with hepatocellular carcinoma after transcatheter arterial chemoembolization. Chin Med J. 2014;127:4204-4209.

13. Zhou D, Liang J, Xu LI, et al. Derived neutrophil to lymphocyte ratio predicts prognosis for patients with HBV-associated hepatocellular carcinoma following transarterial chemoembolization. Oncol Lett. 2016;11:2987-2994. doi:10.3892/ol.2016.4359

14. Rebonato A, Graziosi L, Maiettini D, et al. Inflammatory markers as prognostic factors of survival in patients affected by hepatocellular carcinoma undergoing transarterial chemoembolization. Gastroenterol Res Pract. 2017;2017:4164130. doi:10.1155/2017/4164130

15. Liu C, Jia BS, Zou BW, et al. Neutrophil-to-lymphocyte and aspartate-to-alanine aminotransferase ratios predict hepatocellular carcinoma prognosis after transarterial embolization. Medicine. 2017;96:e8512.

16. Yang X, Zhou S, Wen H, et al. Preoperative neutrophil-lymphocyte ratio as a prognostic predictor after transarterial chemoembolization for HBV-associated hepatocellular carcinoma. Chinese $J$ Radiol. 2015;49:769-773.

17. Chon YE, Park H, Hyun HK, et al. Development of a new nomogram including neutrophil-tolymphocyte ratio to predict survival in patients with hepatocellular carcinoma undergoing transarterial chemoembolization. Cancers. 2019;11:1-12. doi:10.3390/ cancers 11040509

18. Zheng YB, Zhao W, Liu B, Li Y, Hu BS, Lu LG. Prognostic significance of blood neutrophil-to-lymphocyte ratio in patients with hepatocellular carcinoma undergoing TACE. Chinese $J$ Interv Imaging Ther. 2013;10:523-526.

19. Zou W, Chen L, Huang X, et al. The prognostic significance of aspartate aminotransferase to neutrophils ratio in HCC patients treated with TACE. $J$ Interv Radiol. 2017;26:705-711.

20. Huang Z-L, Luo J, Chen M-S, J-q L, Shi M. Blood neutrophil-tolymphocyte ratio predicts survival in patients with unresectable hepatocellular carcinoma undergoing transarterial chemoembolization. J Vasc Interv Radiol. 2011;22:702-709. doi:10.1016/j.jvir.2010.12.041

21. Li JP, Hu SL, Chen H, Bu WZ, Song JL. Blood neutrophil-tolymphocyte ratio predicts survival in patients with unresectable hepatocellular carcinoma undergoing transarterial chemoembolization. Chin J Cancer Pre Treat. 2013;20:522-525. 
22. Sun W, Zhang A, Sui J, Sun D, Wang L, Li W. Neutrophil-tolymphocyte ratio and platelet-to-lymphocyte ratio in prediction of prognosis of patients with hepatocellular carcinoma after TACE. Chinese J Interv Imaging Ther. 2018;15:722-726.

23. Xue T-C, Jia Q-A, Ge N-L, et al. The platelet-to-lymphocyte ratio predicts poor survival in patients with huge hepatocellular carcinoma that received transarterial chemoembolization. Tumor Biol. 2015;36:6045-6051. doi:10.1007/s13277-015-3281-X

24. Fan W, Zhang Y, Wang Y, Yao X, Yang J, Li J. Neutrophil-tolymphocyte ratios as predictors of survival and metastasis for recurrent hepatocellular carcinoma after transarterial chemoembolization. PLoS One. 2015;10:e0119312. doi:10.1371/journal.pone.0119312

25. He C, Zhang Y, Cai Z, Lin X. The prognostic and predictive value of the combination of the neutrophil-to-lymphocyte ratio and the platelet-to-lymphocyte ratio in patients with hepatocellular carcinoma who receive transarterial chemoembolization therapy. Cancer Manag Res. 2019;11:1391-1400. doi:10.2147/CMAR.S190545

26. Schobert I, Savic L, Chapiro J, et al. Neutrophil-to-lymphocyte and platelet-to-lymphocyte ratios as predictors of tumor response in hepatocellular carcinoma after DEB-TACE. Eur Radiol. 2020;30 (10):5663-5673. doi:10.1007/s00330-020-06931-5

27. Yang Z, Zhang J, Lu Y, et al. Aspartate aminotransferase-lymphocyte ratio index and systemic immune-inflammation index predict overall survival in HBV-related hepatocellular carcinoma patients after transcatheter arterial chemoembolization. Oncotarget. 2015;6:43090-43098. doi:10.18632/oncotarget.5719

28. Lu LH, Wei W, Li SH, Zhang YF, Guo RP. The lymphocyte-C-reactive protein ratio as the optimal inflammation-based score in patients with hepatocellular carcinoma underwent TACE. Aging. 2021;13(4):5358-5368. doi:10.18632/ aging. 202468

29. Cruz JC, Watchmaker JM, Albin MM, et al. Neutrophil/lymphocyte ratio predicts increased risk of immediate progressive disease following chemoembolization of hepatocellular carcinoma. J Vasc Interv Radiol. 2019;30(12):1887-1892. doi:10.1016/j.jvir.2019.08.001

30. Craig P, Young S, Golzarian J. Current trends in the treatment of hepatocellular carcinoma with transarterial embolization: variability in technical aspects. Cardiovasc Intervent Radiol. 2019;42 (9):1322-1328. doi:10.1007/s00270-019-02232-7
31. Young S, Craig P, Golzarian J. Current trends in the treatment of hepatocellular carcinoma with transarterial embolization: a cross-sectional survey of techniques. Eur Radiol. 2019;29 (6):3287-3295. doi:10.1007/s00330-018-5782-7

32. Jin J, Zhu P, Liao Y, Li J, Liao W, He S. Elevated preoperative aspartate aminotransferase to lymphocyte ratio index as an independent prognostic factor for patients with hepatocellular carcinoma after hepatic resection. Oncotarget. 2015;6:19217-19227. doi:10.18632/ oncotarget. 4265

33. Hu B, Yang XR, Xu Y, et al. Systemic immune inflammation index predicts prognosis of patients after curative resection for hepatocellular carcinoma. Clin Cancer Res. 2014;20:612-622. doi:10.1158/ 1078-0432.CCR-14-0442

34. Zhao LY, Yang DD, Ma XK, et al. The prognostic value of aspartate aminotransferase to lymphocyte ratio and systemic-inflammation index for overall survival of hepatocellular carcinoma patients treated with palliative treatments. $J$ Cancer. 2019;10(10):2299-2311. doi:10.7150/jca.30663

35. Nagai S, Abouljoud MS, Kazimi M, Brown KA, Moonka D, Yoshida A. Peritransplant lymphopenia is a novel prognostic factor in recurrence of hepatocellular carcinoma after liver transplantation. Transplantation. 2014;97(6):694-701. doi:10.1097/01. TP.0000437426.15890.1d

36. Ding PR, An X, Zhang RX, et al. Elevated preoperative neutrophil to lymphocyte ratio predicts risk of recurrence following curative resection for stage IIA colon cancer. Int $J$ Colorectal Dis. 2010;25 (12):1427-1433. doi:10.1007/s00384-010-1052-0

37. Ohtani H. Focus on TILS: prognostic significance of tumor infiltrating lymphocytes in human colorectal cancer. Cancer Immun. 2007;7:4.

38. Kuang DM, Zhao Q, Wu Y, et al. Peritumoral neutrophils link inflammatory response to disease progression by fostering angiogenesis in hepatocellular carcinoma. J Heaptol. 2011;54(5):948-955. doi:10.1016/j.jhep.2010.08.041

39. Mouchli M, Reddy S, Gerrard M, Boardman L, Rubio M. Usefulness of neutrophil-to-lymphocyte ratio (NLR) as a prognostic predictor after treatment of hepatocellular carcinoma. Ann Hepatol. 2021;22:100249. doi:10.1016/j.aohep.2020.08.067
Journal of Hepatocellular Carcinoma

\section{Publish your work in this journal}

The Journal of Hepatocellular Carcinoma is an international, peerreviewed, open access journal that offers a platform for the dissemination and study of clinical, translational and basic research findings in this rapidly developing field. Development in areas including, but not limited to, epidemiology, vaccination, hepatitis therapy, pathology and molecular tumor classification and prognostication are all considered for publication. The manuscript management system is completely online and includes a very quick and fair peer-review system, which is all easy to use. Visit http://www.dovepress.com/ testimonials.php to read real quotes from published authors. 